\title{
Torascoscopia: una alternativa terapéutica en hemotórax masivo. Informe de un caso y revisión de la literatura
}

\author{
Dr. Paulo Irán Gutiérrez-Torres, ${ }^{1}$ Dr. José Martín Palacios-Acosta, ${ }^{2}$ Dra. Angélica León-Hernández, ${ }^{3}$ \\ Dr. Gilberto Covarrubias-Espinoza, ${ }^{4}$ Dra. Erika F. Sosa-Cruz, ${ }^{5}$ Dr. Jaime Shalkow-Klincovstein ${ }^{6}$ *
}

\begin{abstract}
RESUMEN
La introducción del catéter venoso central (CVC) en pediatría ha significado un gran avance en la medicina moderna y su uso generalizado ha permitido el desarrollo de nuevas técnicas diagnósticas y terapeúticas. El acceso vascular es uno de los procedimientos más frecuente realizados en la actualidad. Se utilizan para monitoreo hemodinámica, hemodiálisis, soporte metabólico y nutricional, administración de líquidos, quimioterapia y antibioticoterapia prolongada, sangre y derivados, y toma de muestras repetidas. Las complicaciones frecuentes incluyen: infecciones, neumotórax, punción arterial y hemotórax. El uso de la toracoscopia para el drenaje de hemotórax secundario es una técnica alternativa útil y segura, con uso de electrocoagulación o bisturí armónico para lograr la hemostasia, que ofrece una recuperación más rápida y menor dolor postoperatorio.
\end{abstract}

Palabras clave: Hemotórax, catéter venoso central, monitoreo hemodinámico, toracoscopia, electrocoagulación.

\section{ABSTRACT}

The use of central venous catheters (CVC) in pediatric patients has implayed a great advance in modern medicine. Their use has allowed the development of new techniques, diagnostic and specialized treatments. Vascular access is one of the most common procedures currently performed by pediatric surgeons. It is used for hemodynamic monitoring, metabolic and nutritional support, fluid administration, chemotherapy, prolonged use of antibiotic, blood transfusion, and samples. The most common complications include: pneumothorax, infections, arterial punction, and hemothorax The use of thoracoscopy for hemothorax drainage, and coagulation of the bleeding vessel with electrocautery or harmonic scalpel, is a useful and safe alternative, which entails faster recovery time and minimal postoperative pain.

Key words: Hemothorax, central venous catheter, hemodynamic monitoring, thoracoscopy, electrocautery.

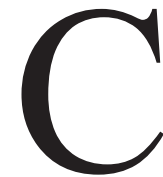

on el desarrollo de novedades terapéuticas en las unidades de terapia intensiva neonatal y pediátrica, la utilización del catéter central, se ha convertido en práctica común en los hospitales de alta especialidad. Los catéteres venosos centrales (CVC) sirven para manejo de grandes volúmenes, como para monitoreó de presión venosa central y alimentación paren-

Jefe de Quirófanos, Anestesia e Inhaloterapia. Hospital Infantil del Estado de Sonora. Reforma 355 Col. Ley 57, C.P. 83100 , Hermosillo, Sonora.

2 Médico adscrito del Servicio de Cirugía Oncológica. Instituto Nacional de Pediatría (INP) drjpalacios@hotmail.com.

3 Pasante de Servicio Social. Servicio de Cirugía Oncológica. INP

4 Jefe del Servicio de Oncología Pediátrica. Hospital Infantil del Estado de Sonora gcovarrubias5@hotmail.com

5 Residente de quinto año de Cardiología Pediátrica. INP erikaflorei1@hotmail.com

6 Jefe del Servicio de Cirugía Oncológica. INP drshalkow@yahoo.com teral; o permanentes, así como la instalación de dispositivos subcutáneos tipo puerto para antibióticos, quimioterapia o toma de muestras. El abordaje de las venas centrales data de principios del siglo XX, cuando en 1927 se utilizó para cateterizar al bulbo superior de la vena yugular interna. Forsman se auto-introdujo un catéter a través de la vena cubital media derecha hasta la aurícula ipsilateral.

* Los autores agradecen al Dr. Shalkow-Klincovstein su participación en la cirugía de este caso.

Correspondencia: Dr. Paulo Irán Gutiérrez-Torres. Hospital Infantil del Estado de Sonora. Reforma 355. Col. Ley 57. Hermosillo, Sonora. CP 83100. Correo electrónico: gutierrezpaulo@hotmail.com Recibido: septiembre, 2009. Aceptado: noviembre, 2009.

Este artículo debe citarse como: Gutiérrez-Torres PI, PalaciosAcosta JM, León-Hernández A y col. Torascoscopia: una alternativa terapéutica en hemotórax masivo. Informe de un caso y revisión de la literatura. Acta Pediatr Mex 2010;31(2):45-49.

www.nietoeditores.com.mx 
Aubaniac en 1952 realiza y describe la técnica de canalización de la vena subclavia por vía infraclavicular por punción percutánea en adultos y posteriormente sería extendido a pacientes pediátricos en la década de los años 80 al 90 del siglo XX. ${ }^{1}$

El acceso subclavio es de elección para el mantenimiento prolongado de un catéter central, por su comodidad y menor riesgo de infecciones asociadas. La incidencia de neumotórax y hemotórax es mayor a la punción por vía yugular con cifras aceptadas como razonables de 1 a $3 \%$, pero en manos experimentadas puede llegar a ser menor de 1/1000. De este modo, la posibilidad de infección, la comodidad del paciente, la facilidad en la fijación y curaciones pasan a ser los factores principales en la decisión de la vía de abordaje. ${ }^{2}$

\section{INFORME DEL CASO}

Niña de cinco años de edad que ingresa al servicio de Oncología Pediátrica con tumor de Wilms derecho estadio II. Se administraron tres ciclos de quimioterapia neoadyuvante como manejo preoperatorio inicial para resección del tumor. Se indicó colocar un catéter venoso central para administrar líquidos, para monitoreo hemodinámico y toma de productos sanguíneos como preparación prequirúrgica. Bajo sedación se colocó el catéter venoso central, en la región subclavia derecha, con la técnica de Seldinger; los primeros tres intentos fallaron; se decidió cambiar la vía de abordaje a través de la vena yugular interna derecha; se tuvo éxito al primer intento. Se tomaron productos sanguíneos y se logró la infusión de líquidos intravenosos.

Inmediatamente después del procedimiento se tomó una radiografía de control, en la que se vio el catéter central y una opacidad derecha que desplazaba estructuras vasculares y desviaba la tráquea hacia el lado izquierdo.

La paciente tenía disnea de medianos esfuerzos, sudación y astenia. Por la sospecha de hemotórax, se decidió colocar una sonda pleural; no hubo mejoría de la sintomatología. El cuadro se agravó; había palidez extrema, diaforesis profusa y frialdad, pulso débil y filiforme, con 140 latidos por minuto y tensión arterial sistólica de 50 mm Hg. El hemitórax derecho estaba abombado e inmóvil, sin murmullo vesicular y mate a la percusión; por la sonda pleural se drenaron 500 cc de líquido hemático. Se trató el estado de choque, se extrajo una muestra de sangre para realizar cruce de hemoderivados y biometría hemática completa, se tomaron radiografias de tórax en serie (Figuras 1,2 y 3). Se observó radiopacidad total del hemitórax afectado compatible con hemotórax masivo.

No hubo mejoría después de colocar la sonda pleural; se decidió recolocar nuevo drenaje; clínica no había me-

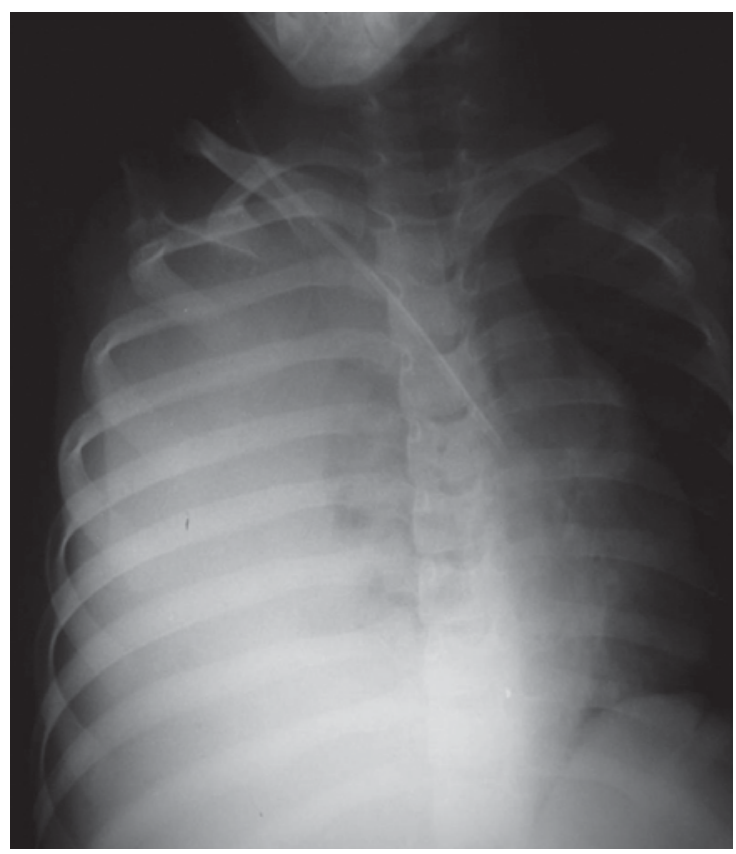

Figura 1. Rx de tórax posterior a la colocación del catéter.

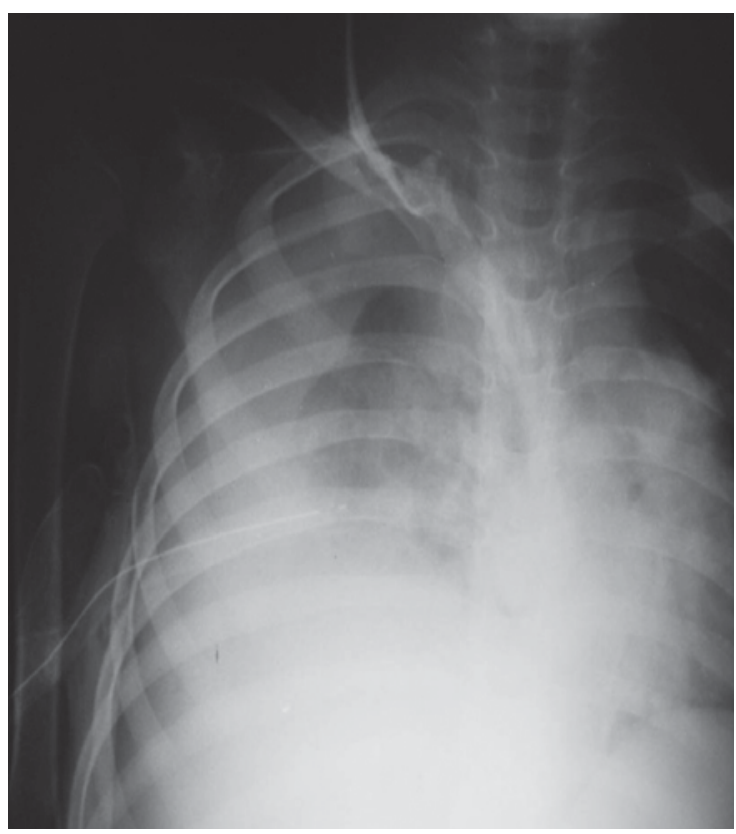

Figura 2. Rx de tórax posterior a la colocación de la sonda pleural (30 min). 


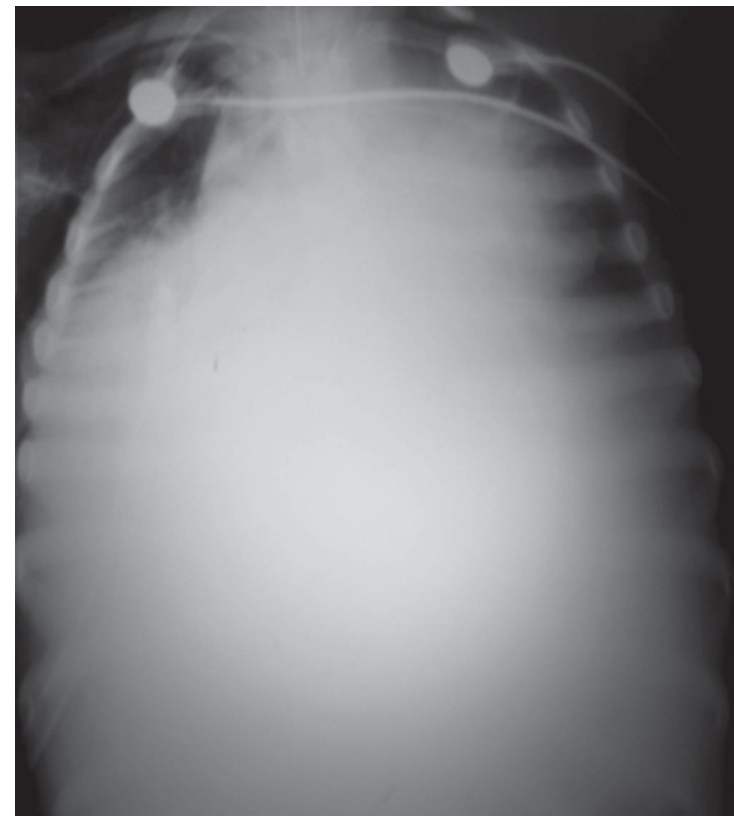

Figura 3. Rx de tórax que muestra opacidad generalizada compatible con hemotórax masivo.

joría. Se decidió la intubación endotraqueal para apoyo ventilatorio, por la sospecha de tórax restrictivo. Se realizó tomografía axial computarizada (TAC) dos horas después, (Figuras 4, 5) mostró hemotórax masivo con probable lesión de la vena subclavia derecha. Se realizó toracoscopia derecha de urgencia.

Se aspiraron $1,500 \mathrm{~mL}$ de sangre aproximadamente y se encontró una lesión longitudinal de $3 \mathrm{~mm}$ en la rama ascendente de la vena subclavia derecha, la cual se electrocoaguló con bisturí armónico laparoscópico y se drenaron abundantes coágulos localizados en la pleura visceral.

Su evolución fue favorable, mejoró hemodinámicamente. Se suturaron los sitios de introducción de los puertos de $5 \mathrm{y}$ $10 \mathrm{~mm}$, y se dejó un drenaje pleural hacia la periferia y cerca del ángulo costofrénico, se conectó para aspiración central.

El procedimiento se realizó sin complicaciones. Se tomó RX de tórax de control que no mostró evidencia de hemotórax, había expansión pulmonar; se extubó a las $24 \mathrm{~h}$ después del procedimiento quirúrgico. Evolucionó satisfactoriamente, sin deterioro pulmonar.

\section{DISCUSIÓN}

La cateterización intravascular venosa o arterial, está indicada con fines diagnósticos y terapéuticos. La colocación de un $\mathrm{CVC}$ tiene indicaciones y algunas complicaciones.

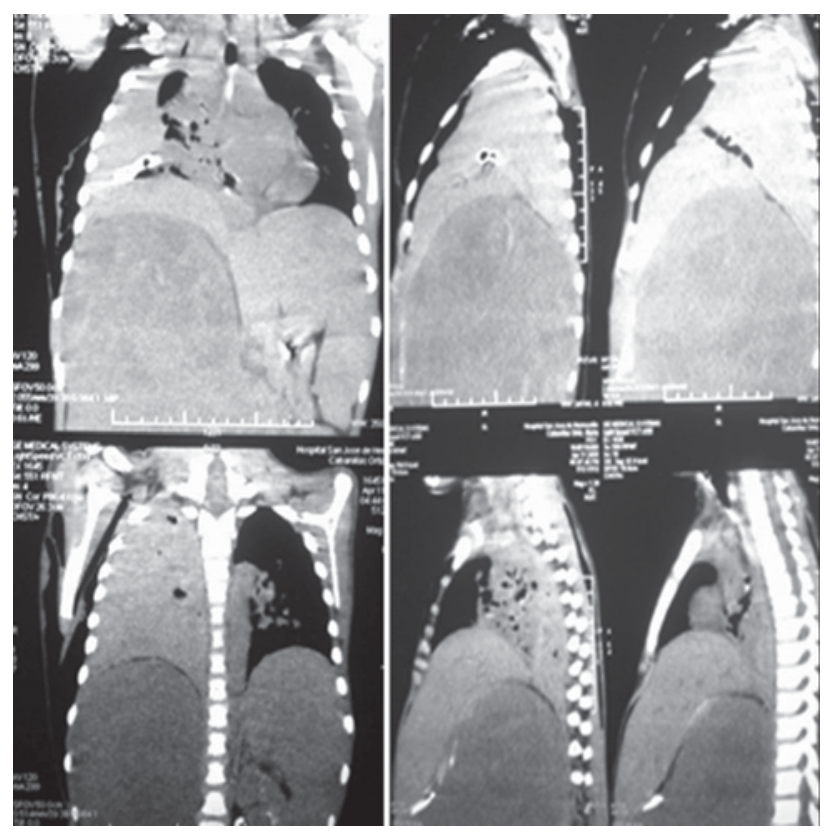

Figura 4. Tomografía axial computarizada que muestra hemotórax masivo con desplazamiento de la tráquea y estructuras vasculares.

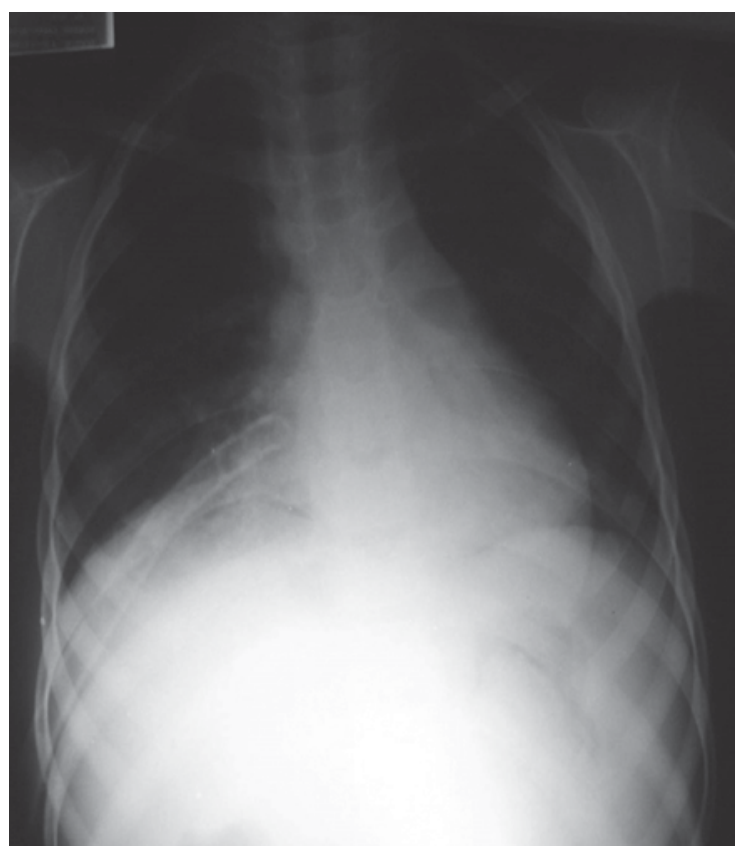

Figura 5. Rx de tórax de control posterior al drenaje del hemotórax.

\section{COMPLICACIONES DE LA COLOCACION DEL CATÉTER VENOSO CENTRAL POR PUNCIÓN}

Se han enumerado más de 38 complicaciones, mecánicas, técnicas o infecciosas relacionadas con el catéter venoso 
central. Las primeras generalmente se relacionan con la inserción del catéter y según su gravedad, se clasifican en mayores o menores. Son complicaciones mayores, que ponen en riesgo la vida del paciente como el neumotórax, hidrotórax, quilotórax, hemotórax, la fístula arteriovenosa, el desgarro de la vena, la punción carotídea. Las menores son la dehiscencia de la herida, exposición del catéter etc. Las complicaciones infecciosas, también pueden comprometer la vida del paciente, incrementan el tiempo de hospitalización y los costos hospitalarios.

Entre los factores que se asocian a las complicaciones están el sitio de inserción o de punción, la lesión vascular, dislocación o movilización del catéter, la perforación de estructuras vecinas al sitio de la punción, etc. ${ }^{7}$ Para detectar las complicaciones tempranas o tardías se debe tener en consideración que una radiografía de tórax normal no descarta las complicaciones tardías; se debe hacer ésta para confirmar la situación del catéter en el momento de la inserción y revisarla siempre que se realice una radiografía por otra causa.

La laceración de estructuras vasculares puede asociarse con hematomas, especialmente en pacientes con alteraciones de la coagulación. El embolismo aéreo es una complicación infrecuente pero que puede conducir a arritmias, infarto del miocardio, endocarditis, embolismo pulmonar y cerebral con sus manifestaciones clínicas y secuelas, incluso a la muerte en un alto porcentaje de estos pacientes.

Los derrames pleurales tardíos secundarios a cateterización de la subclavia pueden tener una incidencia menor del $0.5 \%$. El derrame pleural contralateral es una complicación rara posterior a la cateterización de la vena subclavia y casi siempre de aparición tardía, se acompaña de erosión vascular. Las erosiones vasculares crónicas debidas a la acción del catéter que actúa como cuerpo extraño, producen hidrotórax. Probablemente por la perforación vascular gradual que permite el establecimiento de la hemostasia alrededor del catéter en el lugar de la perforación. La composición del hidrotórax sería similar a la de la solución intravenosa infundida por el catéter. ${ }^{8}$

Las causas que pueden provocar la perforación del vaso en el hemotórax deben dividirse en función del momento en que se produzca, es decir, una perforación inmediata o tardía. En el primer caso se asocia a una punción directa del vaso o al uso de guías o catéteres demasiado rígidos. Las perforaciones tardías son extraordinariamente raras, ocurren entre las 24 horas y el séptimo día de colocación, por erosión progresiva de la pared del vaso. ${ }^{9,10}$

\section{CAUSAS DE PERFORACION PLEURAL}

1. Cabalgamiento de la punta del catéter entre la cavidad pleural y la luz del vaso; los líquidos perfundidos fluirán hacia la cavidad pleural por presión negativa.

2. La punción pleural y vascular inmediata genera un pequeño hemotórax que al ser aspirado, confunde sobre la colocación intravascular.

3. La punta del catéter erosiona la pared del vaso, que con la acumulación de líquido irritante y los movimientos cardiorrespiratorios y posturales del paciente erosionan el vaso, lo que causa la difusión del líquido al espacio pleural.

En un paciente con traumatismo torácico y hemotórax, en el que sea necesario canalizar una vía central, no se aconseja canalizar la vía del lado del traumatismo, si se sospecha la fractura de la primera costilla, pues se ha descrito la posibilidad de puncionar un hemotórax no detectado previamente por radiología, lo que favorece la colocación inadecuada del CVC, y otras complicaciones potencialmente graves. En caso de hemotórax, el reflujo de sangre por el catéter no es un signo fiable de la localización intravascular del catéter, se requieren otros métodos para comprobar su colocación correcta. ${ }^{11,12}$

Un error frecuente es comprimir el orificio cutáneo de salida cuando hay salida de sangre tras la inserción. El punto a comprimir es la zona de la punción venosa, en la fosa supraclavicular, aunque lo más efectivo es evitar el decúbito manteniendo al paciente sentado para reducir la presión venosa central.

Una infusión de desmopresina $(0.3 \mu \mathrm{g} / \mathrm{kg}$ en $20 \mathrm{minu}-$ tos) puede mejorar la hemostasia el tiempo suficiente para controlar el sangrado postinserción. ${ }^{13,14}$

\section{USO DE TORACOSCOPIA EN HEMOTÓRAX SECUNDARIO A LESIÓN VASCULAR}

Branco en 1946 realizó la primera toracoscopia para evaluar la extensión del daño torácico luego de lesiones torácicas penetrantes; a través de esta vía solucionó sangrados de origen arterial y venoso. ${ }^{15}$ En la última década del siglo XX el uso de la cirugía torácica video asistida (CTVA), se extendió debido al rápido desarrollo de los 
equipos de videocirugía, con lo cual se mejoró la calidad en la atención del paciente critico. ${ }^{16,17}$ En México comenzó a usarse como toracoscopia diagnóstica en los años 80 y en 1990 se introdujo la cirugía video toracoscopia con fines terapéuticos; en el año 2000, se inició el uso de la cirugía de mínima invasión en trauma vascular, pero en adultos.

La toracoscopia persigue tres objetivos:

- El control de la fuente de hemorragia.

- La evacuación de la cavidad pleural.

- La reexpansión pulmonar.

\section{CONCLUSIONES}

Las lesiones vasculares como complicaciones relacionadas con la inserción de catéteres venosos subclavios infrecuentes. El hemotórax ocurre en un porcentaje menor al 1\% y en algunos casos con resultados fatales. La detección de esta complicación se basa en la clínica y teniendo en cuenta esta posibilidad como diagnóstico. Se podrá introducir medio de contraste hidrosoluble sobre el catéter y verificar radiológicamente si existen fugas del mismo o hay dirección anómala.

El tratamiento del trauma vascular torácico secundario a la colocación del catéter venoso central por la técnica de Seldinger incluye tres aspectos esenciales e inviolables que son: Una evaluación inmediata de la magnitud de la lesión, clínica y hemodinámica si el estado del paciente lo permite. Una actuación integrada, consciente y urgente en el control de la hemorragia, la reposición de volumen y la corrección de la causa. Además de la elección adecuada de la técnica quirúrgica por toracotomía, por mínima invasión o por ambas, así como el personal profesional con experiencia que tomará conductas y decisiones en el tratamiento de este tipo de lesiones. ${ }^{1-3}$ Solo teniendo en cuenta estos principios se logrará aumentar la sobrevida de los pacientes que sufren un trauma vascular torácico secundario al uso de acceso vascular central. Puesto que algunos pacientes con estas lesiones pueden progresar hacia una resolución fatal en horas, el éxito del tratamiento constituye un reto para el cirujano pediatra. Obtener y formar los recursos humanos y tecnológicos, permitirá tratar adecuadamente una urgencia torácica pediátrica mortal. ${ }^{15,16}$

\section{REFERENCIAS}

1. Rollo J, Campistol J, Almirall J, Cases A, Montolin J, Revert L. Complicaciones precoces asociadas a la cateterización de la vena subclavia como acceso vascular para hemodiálisis. Med Intensiv. 1988;12(2):85-8.

2. David C, McGee M. Preventing complications of central venous catheterization. Engl J Med. 2003;348:12-20.

3. Paul F, Mansfield D, Bruno H, Fornage D. Complications and failures of subclavian-vein catheterization. N Engl J Med. 1995;332:1579-81.

4. Wallace M, Ahrar K. Percutaneous closure of a subclavian artery injury after inadvertent catheterization. J Vasc Interv Radiol. 2001;12:1227-30.

5. Oakes D, Wilson R. Malposition of a subclavian line. Resultant pleural effusions, interstitial pulmonary edema, and chest wall abscess during total parenteral nutrition. JAMA. 1975;233:5323.

6. Curtis L, Timothy A, Burke D, Cardela J, Steven J, Cole P, Drooz A, et al. Quality improvement guidelines for central venous access. J Vasc Interv Radiol. 2003;14:231-5.

7. Merrer J, De Jonghe B, Golliot F. Complications of femoral and Subclavian venous catheterization in critically ill patients: a randomized controlled trial. JAMA. 2001;286:700-7.

8. Rodríguez J, Bárcena M, Álvarez J. Hemotórax agudo contralateral tras la canulación de la vena subclavia izquierda para hemodiálisis. Rev Esp S Anestesiol Reanim. 2002;49:428-43.

9. Mukau L, Talamini M, Sitzmann J. Risk factors for central venous catheter related vascular erosions. J Parenter Enteral Nutr. 1991;15:513-68.

10. Krauss D, Schmidt GA. Cardiac tamponade and contralateral haemothorax after subclavian vein catheterization. Chest. 1991;99:517-8.

11. Del Castillo. El reflujo de sangre por el catéter al intentar canalizar la vena subclavia no es un signo fiable en caso de hemotórax. Rev Esp Anestesiol. 2005;5:51-5.

12. Niño JL, Sánchez R. ¿Cuál es el mejor momento para el drenaje por toracoscopía del hemotórax coagulado? Panam J Trauma. 2006;13(2):51-6.

13. Díaz C, Mérida A, Minero J. Hemotórax traumático retenido manejado con toracoscopía. Trauma. 2007;10(2):3740.

14. Grinspan R. Traumatismo torácico y toracoabdominal. Rol de la Videocirugía. Rev Arg Cirug. 1999;76:25361.

15. Garzón J. Indicaciones y contraindicaciones actuales para cirugía toracoscópica. Mc Graw Hill; 2007.

16. Rodríguez F. Toracoscopía: presente y futuro. Pneuma. 2005;(Suplemento 1):7-10.

17. Andrade R, Pérez G, Ávila A. Toracoscopía rígida en el manejo del hemotórax traumático coagulado. Rev Inst Nac Enferm Resp 2005;3:1958.

18. García N, Núñez $O$, Pasini R, Hernández L, Sánchez J. Toracoscopía en trauma. Indicaciones, resultados y protocolo institucional de aplicación en el Hospital Central Militar. Asociación Mexicana de Cirugía Endoscópica, A.C.; 2007. p. 60-66. 